\title{
Imaging and Spectroscopy of Magellanic Cloud Planetary Nebulae obtained with the Hubble Space Telescope
}

\author{
E. Vassiliadis ${ }^{1}$, M. Dopita ${ }^{2}$, R.C. Bohlin ${ }^{3}$, J.P. Harrington ${ }^{4}$, \\ S.J. Meatheringham ${ }^{2}$, H.C Ford ${ }^{5}$, P.R. Wood ${ }^{2}$, T.P Stecher ${ }^{6}$ and S.P. Maran ${ }^{6}$ \\ ${ }^{1}$ Instituto de Astrofísica de Canaria, Spain; \\ ${ }^{2}$ Mount Stromlo \& Siding Spring Observatories, Australia; \\ ${ }^{3}$ Space Telescope Science Institute, USA; ${ }^{4}$ University of Maryland, USA; \\ ${ }^{4}$ University of Maryland, USA; ${ }^{5}$ Johns Hopkins University, USA; \\ ${ }^{6}$ Goddard Space Flight Centre, USA.
}

The majority of planetary nebulae (PNe) at the distance of the Magellanic Clouds typically subtend 1 arcsec or less on the sky. The Planetary Camera onboard the Hubble Space Telescope has a scale of 0.044 arcsec per pixel and is therefore ideal to use to image these objects. At present, 16 LMC and 5 SMC objects have been imaged in the [O III] 5007 angstrom emission line, as part of this program. An additional 10 LMC and 5 SMC objects have been imaged in the same line under the Guaranteed Time Observer program.

A wealth of information from ground-based observatories exists for this sample, including optical spectrophotometry, absolute H-Beta photometry, and nebular [O III] expansion velocities (see Dopita, these proceedings). However, one deficiency is the lack of any information about $\mathrm{C}$, an important element in AGB evolution and nebular photoionization.

The Faint Object Spectrograph is used to obtain spectra from 1150 to 3600 angstroms for most of the sample, to complement the existing ground-based spectrophotometry from 3600 to 8000 angstroms. In addition to measuring important $C$ lines, the far ultraviolet spectrum is dominated by stellar emission, enabling the stellar temperature to be determined directly.

The combined HST and ground data are used to compute new nebular photoionization models. The results allow reliable placement of the PN nuclei on the HR diagram, and the study of the relative $\mathrm{C}, \mathrm{N}$, and $\mathrm{O}$, abundances with respect to evolution along the AGB.

HST time has been allocated to obtain images and spectra for an additional fifteen objects in the Large Magellanic Cloud. 